\title{
Analysis of Heat Transfer and Fluid Flow in the Continuous Casting Mold with Electromagnetic Brake
}

\section{Kouji TAKATANI, Ken NAKAI, Norifumi KASAl, Tadao WATANABE') and Hidemasa NAKAJIMA ${ }^{1)}$}

Iron and Steel Research Laboratories, Sumitomo Metal Industries, Ltd., Oaza Sunayama, Hasaki-machi, Kashima-gun, Ibaraki-ken, 314-02 Japan. $\quad$ 1) Kashima Steel Works, Sumitomo Metal Industries, Ltd., Oaza Hikari, Kashima-machi, Kashima-gun, Ibaraki-ken, 314 Japan.

(Received on February 14, 1989; accepted in the final form on May 19, 1989)

\begin{abstract}
A three-dimensional mathematical model has been developed to analyze the heat transfer and the fluid flow in the continuous casting mold with electromagnetic brake. For verification of the mathematical model, the comparison of the flow field between the experimentally measured and computed results has been done under the isothermal condition.

The theoretical predictions of the temperature field and heat transfer phenomena are compared and discussed with experimental results.
\end{abstract}

KEY WORDS: continuous casting; heat transfer; fluid flow; electromagnetic field; mathematical model.

\section{Introduction}

It is very important to understand the heat transfer and the fluid flow in the continuous casting mold for the quality of products.

In recent years, there has been a considerable interest in the study of electromagnetic brake (EMBR) ${ }^{1 \prime}$ adopted to suppress the deep penetration of molten steel flow in the continuous casting mold.

As a result of experimental and computational work in this field, ${ }^{2,3}$ the fluid flow with electromagnetic phenomena in the continuous casting mold is well understood. However, it is not sufficient to analyze the heat transfer and the fluid flow simultaneously by the mathematical model with the limitation of the ability of the computer.

In this paper, simultaneously we analyze the heat transfer and the fluid flow with EMBR in the continuous casting mold by means of the super-computer.

\section{Formulation}

\subsection{Governing Equations}

The following assumptions and simplifications are made in this model:

(1) The fluid is incompressible and the Boussinesq approximation ${ }^{4}$ is applied for non-isothermal condition.

(2) Electromagnetic characteristics of fluid are uniform and isotropic.

(3) The density and the specific heat are taken to be same value for solid and liquid phase.

(4) The velocity of solid phase is equal to the casting speed. $\left(\mathbf{U}_{s}=\mathbf{V}_{c}\right)$

(5) The velocity of liquid phase is equal to the velocity of solid phase for $f_{s}>0.8$.

The governing equations take the following form:

$$
\begin{aligned}
& \nabla \cdot\left(f_{s} \mathbf{U}_{s}+f_{l} \mathbf{U}_{l}\right)=0 \\
& \frac{\partial \mathbf{U}_{l}}{\partial t}+\left(\mathbf{U}_{l} \cdot \nabla\right) \mathbf{U}_{l} \\
& =\frac{1}{\rho}\left[-\nabla P+\nabla\left(\mu \nabla \cdot \mathbf{U}_{l}\right)+\beta\left(T_{0}-T\right) \rho \mathbf{g}+\mathbf{F}\right] \\
& \frac{\partial T}{\partial t}+\nabla \cdot\left(f_{s} T \mathbf{U}_{s}\right)+\nabla \cdot\left(f_{l} T \mathbf{U}_{l}\right) \\
& =\frac{1}{\rho C_{p}}[\nabla(k \nabla T)+\rho \operatorname{Rate}(-\Delta H)] \\
& \frac{\partial f_{s}}{\partial t}+\nabla \cdot\left(f_{s} \mathbf{U}_{s}\right)=\text { Rate }
\end{aligned}
$$

where, $\mathbf{F}=\mathbf{J} \times \mathbf{B}$

Rate: Rate of solidification.

Furthermore, the MHD approximation ${ }^{51}$ is applied. The governing equations of electromagnetic field take the following form:

$$
\begin{aligned}
& \nabla \cdot \mathbf{J}=0 \\
& \mathbf{E}=-\nabla \phi \quad \ldots \ldots \ldots \\
& \mathbf{J}=\sigma\left(\mathbf{E}+\mathbf{U}_{l} \times \mathbf{B}\right)
\end{aligned}
$$

We obtained the following Poisson equation of the electric potential $\phi$.

$$
\nabla(\sigma \nabla \phi)=\nabla \cdot \sigma\left(\mathbf{U}_{l} \times \mathbf{B}\right)
$$

In order to determine the temperature field and the flow field, it is necessary to solve the Eqs. (1) to (4) and (8) under the proper boundary conditions.

\subsection{Boundary Conditions}

Fig. I shows the boundary conditions of the flow field, the heat transfer and the electric field.

\subsection{Method of Solution}

\subsubsection{Grid System}

For a three-dimensional calculation, the flow re- 


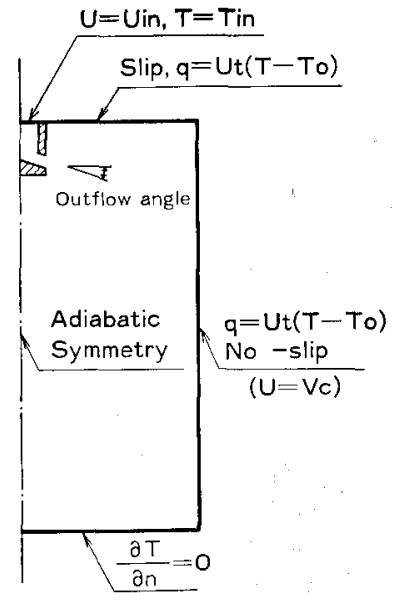

a) flow and thermal condition

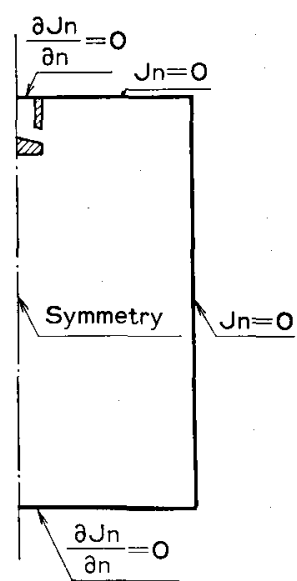

b) electrical condition
Fig. 1. Boundary condition.

gion is subdivided into a set of the hexahedron cells as shown in Fig. 2. For each center of cell surface velocity $U^{*}, V^{*}$ and $W^{*}$ which are normal to each cell surface are stored. Pressure, temperature and fraction solid are stored for the center of each cell. When the coordinate system $(\xi, \eta, \zeta)$ is not orthogonal, it is necessary to transform the governing equations as is mentioned in the latter.

\subsubsection{The Finite-difference Form}

According to the Gauss' divergence theorem,, ,7) the partial derivatives $\partial f / \partial x, \partial f / \partial y$ and $\partial f / \partial z$ can be written in the following form:

$$
\left.\begin{array}{l}
V_{m} \frac{\partial f}{\partial x}=\left(S_{\xi x} f\right)_{\xi}+\left(S_{\eta x} f\right)_{\eta}+\left(S_{\zeta x} f\right)_{\zeta} \\
V_{m} \frac{\partial f}{\partial y}=\left(S_{\xi y} f\right)_{\xi}+\left(S_{\eta y} f\right)_{\eta}+\left(S_{\xi y} f\right)_{\xi} \\
V_{m} \frac{\partial f}{\partial z}=\left(S_{\xi z} f\right)_{\xi}+\left(S_{\eta z} f\right)_{\eta}+\left(S_{\xi z} f\right)_{\xi}
\end{array}\right\}
$$

where, $S_{l m}$ : Projection area of cell surface $l$ to the direction $m(l=\xi, \eta, \zeta, m=x, y, z)$

$V_{m}$ : Volume of cell.

\subsubsection{Transformation of Governing Equations}

The velocity $\left(U^{*}, V^{*}, W^{*}\right)$ are normal to the cell surfaces as shown in Fig. 1. Next equations are developed by writing a mass balance over a cell surface through which the fluid is flowing.

$$
\left.\begin{array}{l}
S_{\xi} U^{*}=S_{\xi x} U+S_{\xi y} V+S_{\xi z} W \\
S_{\eta} V^{*}=S_{\eta x} U+S_{\eta y} V+S_{\eta z} W \\
S_{\xi} W^{*}=S_{\zeta x} U+S_{\zeta y} V+S_{\zeta z} W
\end{array}\right\}
$$

where, $S_{l}$ : Area of cell surface $(l=\xi, \eta, \zeta)$.

It is possible to transform from $(U, V, W)$ to $\left(U^{*}\right.$, $\left.V^{*}, W^{*}\right)$ and from $\left(U^{*}, V^{*}, W^{*}\right)$ to $(U, V, W)$ by the use of Eq. (10).

For example, the divergence of vector $\mathbf{U}$ is transformed:

$$
\nabla \cdot \mathbf{U}=\frac{1}{V_{m}}\left[\left(S_{\xi} U *\right)_{\xi}+\left(S_{\eta} V^{*}\right)_{\eta}+\left(S_{\xi} W^{*}\right)_{\xi}\right]
$$

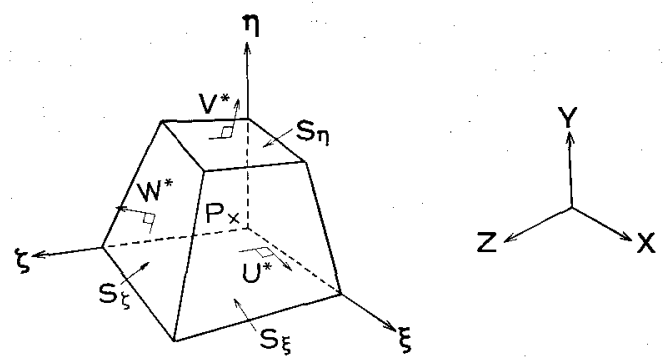

Fig. 2. Grid system.

Similarly, Eqs. (1) to (4) and (8) are transformed. (omitted in detail)

\subsection{Numerical Procedure}

The technique adopted in the present case was the use of SOLA ${ }^{8)}$ method for the solution of the equation of motion and continuity. And the time integration method of the equation of energy conservation was the temperature recovery method, ${ }^{9,10)}$

Numerical procedure can be summarized as follows:

(1) The body force term $\mathbf{F}=\mathbf{J} \times \mathbf{B}$ is obtained from the solution of

$$
\nabla(\sigma \nabla \phi)=\nabla \cdot \sigma\left(\mathbf{U}_{l} \times \mathbf{B}\right)
$$

(2) Velocity and pressure of the next time step $U_{1}^{n+1}, P^{n+1}$ are obtained by SOLA method. The time integration is semi-implicit.

(3) Tentative temperature $\tilde{T}$ and rate of solidification Rate are obtained by following equations.

$$
\begin{aligned}
& \frac{\tilde{T}-T^{n}}{\Delta t}+\nabla \cdot\left(f_{s} \mathbf{U}_{s}\right)+\nabla \cdot\left(f_{l} \mathbf{U}_{l}\right)={ }_{\rho} \frac{1}{\rho C_{p}} \nabla(k \nabla T) \\
& \text { Rate }=C_{p}(T-\tilde{T}) /(-\Delta H \cdot \Delta t), T=T_{t}-\left(T_{l}-T_{s}\right) f_{s}
\end{aligned}
$$

(4) Temperature and fraction solid of the next time step $T^{n+1}, f_{s}^{n+1}$ are obtained by the temperature recovery method. The time integration is implicit.

(5) Above-stated procedure is repeated until no cell exhibits a temperature change.

$$
\left|\frac{T^{n+1}-T^{n}}{T^{n+1}}\right|<10^{-4}
$$

The time interval $\Delta t$ is set as

$$
\frac{U_{l} \cdot \Delta t}{\Delta l}=0.1,
$$

where, $\Delta l: \quad$ A length of the cell.

It takes about $0.3 \mathrm{~s} / \mathrm{cycle}$ for non-isothermal condition by means of the super computer NEC SX-2 system.

\section{Results and Discussion}

\subsection{Comparison of Measured and Computed Results}

For verification of the computational technique mentioned above, the comparison of the flow field between the experimentally measured and computed results has been done.

Fig. 3 shows a schematic view of the cold model 
which is the scale of $1 / 2.5$ of No. 3 continuous casting machine at Kashima Steel Works. The velocity at $15 \mathrm{~mm}$ below the meniscus was measured by the propeller flowmeters and the influence of the outflow angle of immersion nozzle and flow rate were investigated. The equality of the Froude number is adopted in order to determine the experimental conditions.

\subsubsection{Finite Difference Grid}

Fig. 4 shows the region of analysis and the grid (31-cell-long, 27-cell-width and 15-cell-thickness) for the computational analysis.

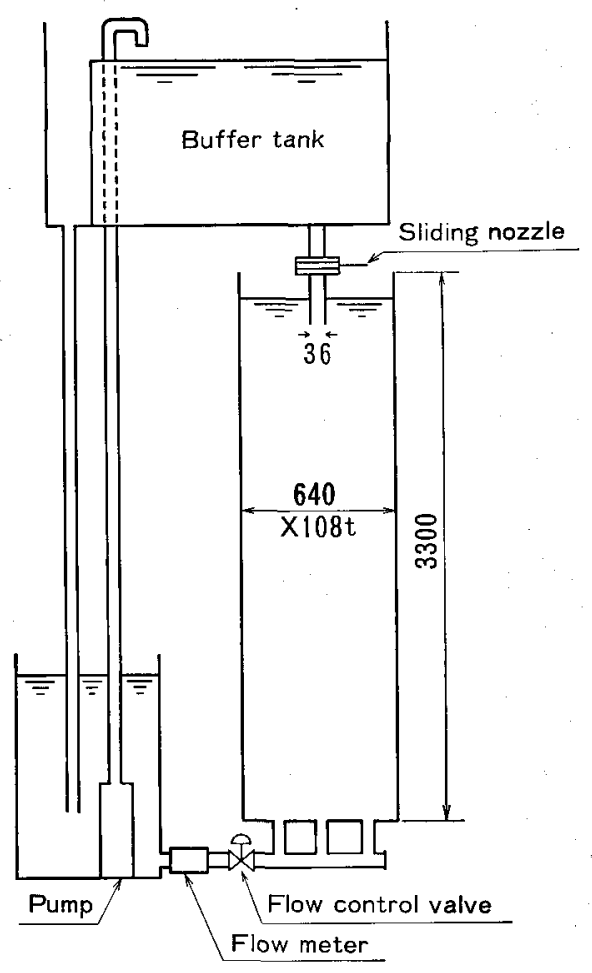

Fig. 3. Experimental apparatus.
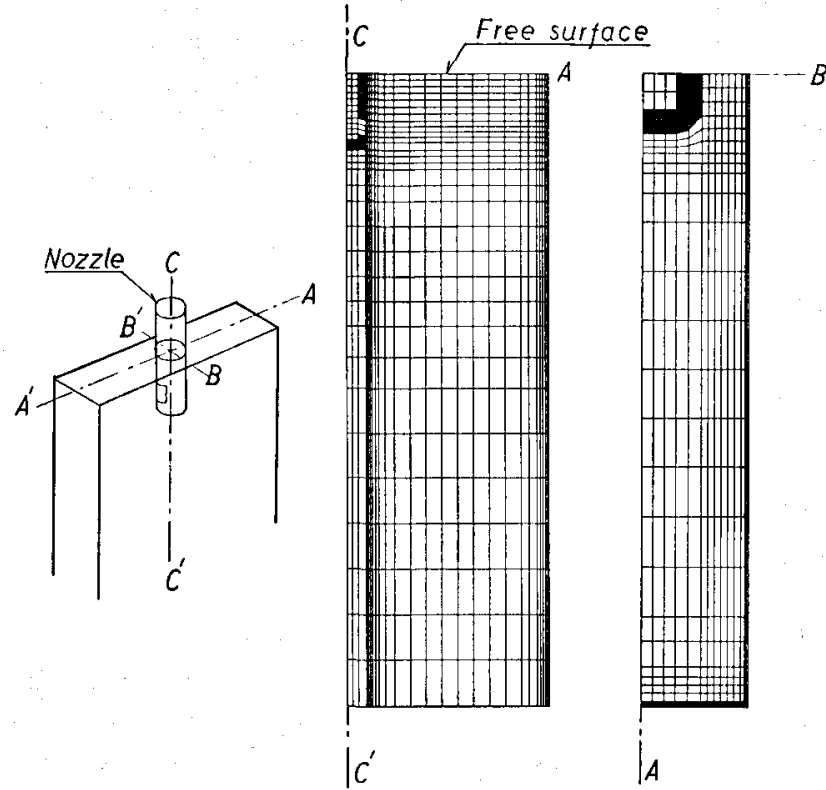

Fig. 4. Computational region and grid.

\subsubsection{Effect of the Outflow Angle of Immersion Nozzle}

The convection term of the equation of motion was adopted the first-order upwind scheme and the boundary condition at the mold was a no-slip condition. Fig. 5 shows the comparison of the velocity at $15 \mathrm{~mm}$ below the meniscus between measured and computed results. There is good agreement between measured and computed results.

The fluid flows along the direction of the outflow angle of the immersion nozzle near the outlet of the nozzle, but the whole fluid flow is not different so much according to the outflow angle.

\subsubsection{Effect of Flow Rate (Casting Speed $V_{c}$ )}

Fig. 6 shows a effect of flow rate through the immersion nozzle. With increasing flow rate the velocity is faster at the meniscus. Here again, there is quantitative agreement between the measurements and the predictions.

The agreement appears to be quantitative without any kind of turbulent model by means of dividing the grid into small parts near the mold wall.

In the calculations with coarse grid, the maximum velocity at the meniscus is low as compared with the expcrimental data, but becomes to be closer to the measured value with the finer grid near the mold wall.

In this study, changing with the passage of time on the fine flow structure is not reproduced but the timeaveraged flow field can be simulated with the fine
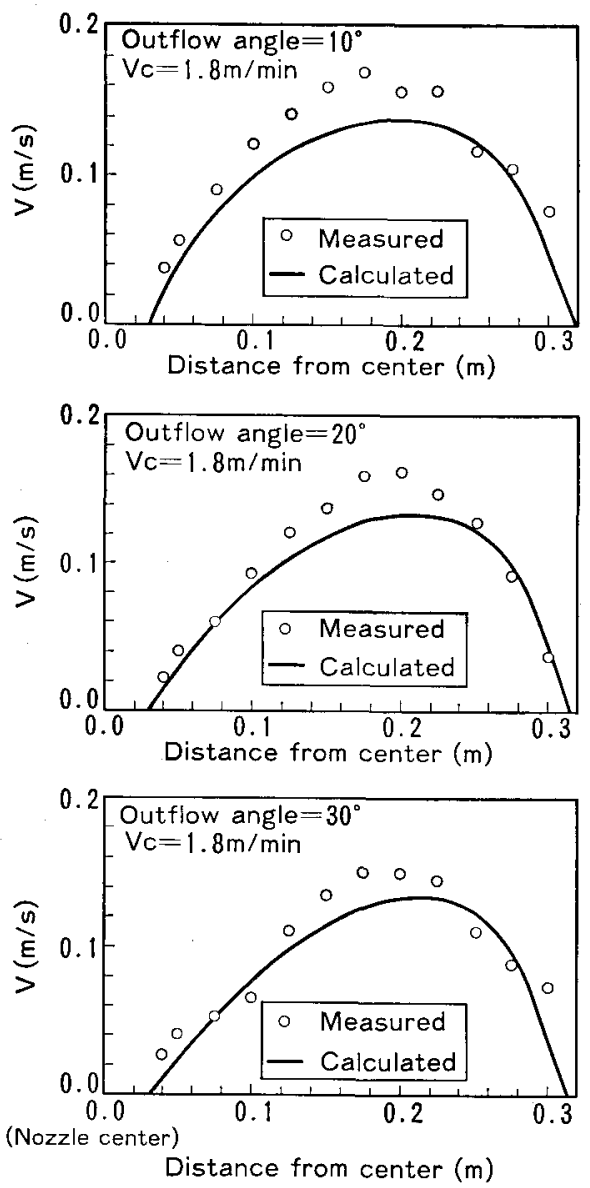

Fig. 5. Effect of outflow angle on velocity at $15 \mathrm{~mm}$ below the meniscus. 

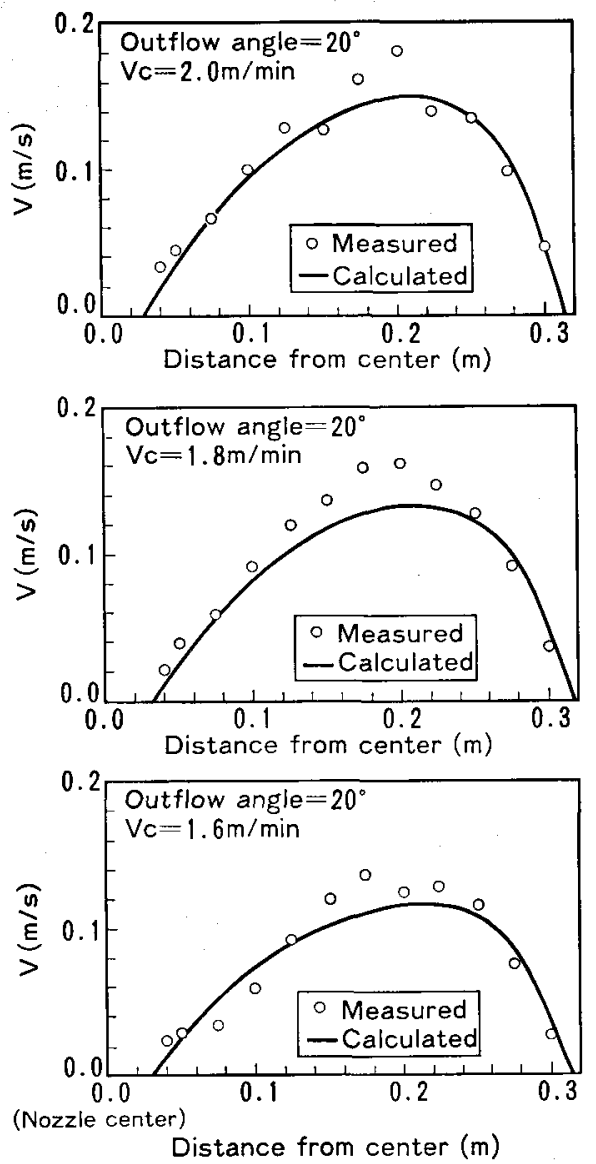

Fig. 6. Effect of casting speed on velocity at $15 \mathrm{~mm}$ below the meniscus.

grid which reduces the artificial diffusion near the mold wall.

\subsection{Isothermal Analysis}

In order to clarify the characteristics of EMBR, the qualitative effect of EMBR on the flow field was analyzed with a coarse grid under the isothermal condition.

\subsubsection{Computational Grid and Distribution of the Magnetic Flux Density}

Fig. 7(a) shows the grid for the computational analysis and Fig. 7(b) shows the magnetic flux density. The distribution of magnetic flux density was fitted in the measurements.

\subsubsection{Effect of the Intensity of the Magnetic Flux} Density on the Flow Field

Fig. 8 shows the effect of the intensity of the magnetic flux density on the flow field. When the maximum value of the magnetic flux density is over about 0.17 Tesla, upper circulation flow is affected and there are two circulation loops at 0.35 Tesla.

Constant flow rate can be controlled by the opening of the sliding nozzle, since the electromagnetic force in the immersion nozzle is very small.

\subsubsection{Effect of the Position of EMBR}

Ordinally two couples of electromagnet are placed between the immersion nozzle and the narrow side of

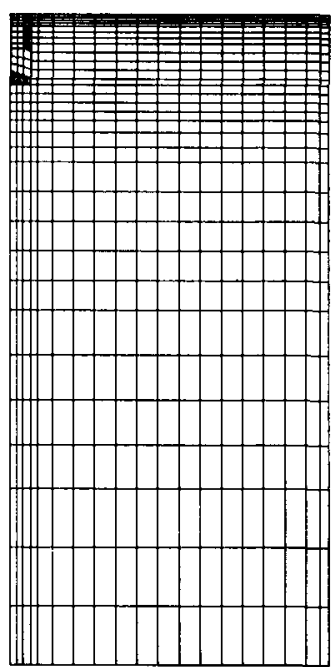

a) Grid

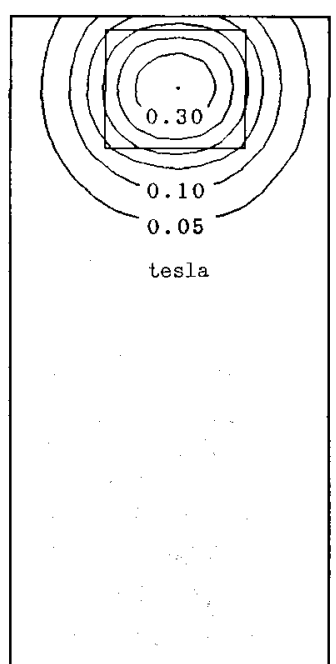

b) Magnetic flux density
Fig. 7. Computational grid and magnetic flux density.

the mold, but it may be possible to control the fluid flow by a couple of electromagnet. It is able to control the fluid flow by a couple of electromagnet in the same way as shown in Fig. 9.

If the fluid flow can be controlled by a couple of electromagnet, consumption of electric power that creates the electromagnetic field is small and it may be possible to reduce the total weight of the mold, electric power for oscillation of the mold can be low.

\subsection{Non-isothermal Analysis}

In this section, we examine the effects of the EMBR on the heat transfer and the fluid flow under the nonisothermal condition. The characteristics of molten steel and the heat transfer coefficient at free surface, mold and spray region are shown in Tables 1 and 2 .

Fig. 10 shows the comparison of the temperature profile at $30 \mathrm{~mm}$ below the meniscus between measured and computed results and Fig. 11 shows the temperature profile at $1 / 2$ thickness of the slab and the meniscus. It can be seen that the temperature near the meniscus is higher at $1 / 2$ thickness of the slab with EMBR than without EMBR but the temperature gradient at the meniscus is steeper with EMBR than without EMBR. The fluid flow has a strong circulating flow below the meniscus without EMBR. On the other hand, the fluid flow through the immersion nozzle is curved upward and downward by EMBR and the circulating flow is weak near the meniscus. It may be said that the fluid flow controls the heat transfer phenomena in the continuous casting mold.

It is possible to suppress the deep penetration of molten steel flow and accelerate a rising of inclusions and to preserve the break-out from remelting of the solidified shell at the narrow side of the mold with EMBR. The other hand, since the temperature gradient near the meniscus is steeper with EMBR than without EMBR, when the distribution of magnetic flux density is not appropriate, melting rate of the powder becomes to be uneven and it is no good 
Fig. 8.

Effect of the intensity of the magnetic flux density on the flow field. (at 1/4 thickness of the slab)

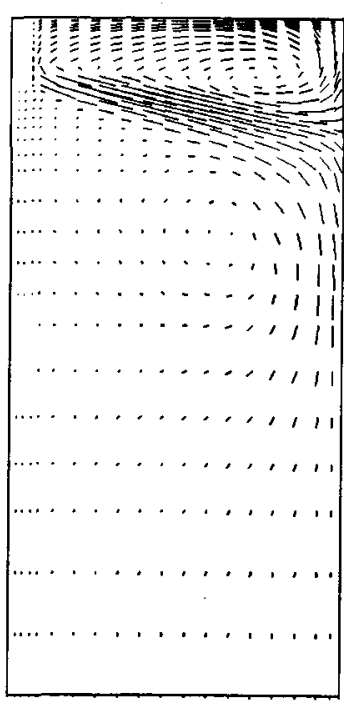

$\operatorname{Bmax}($ tesla $)=0.0$

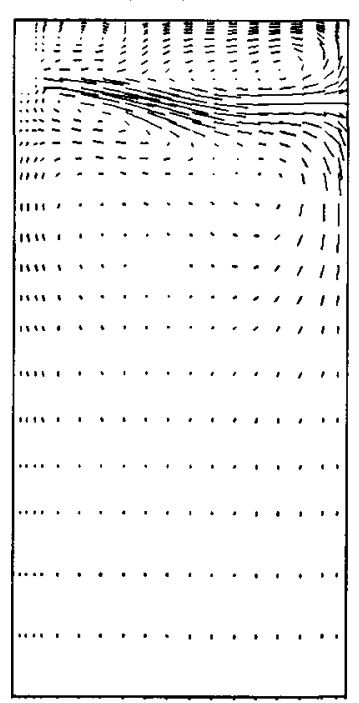

0.17

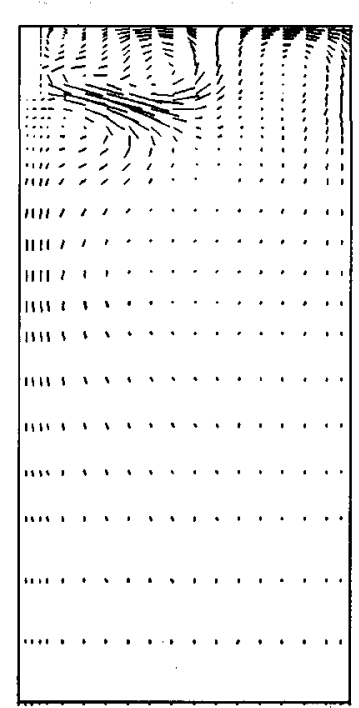

0.35

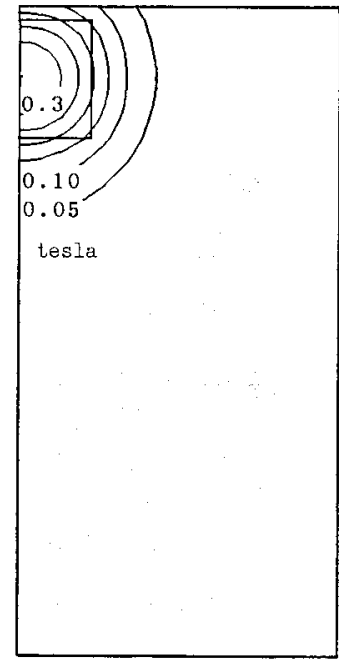

Magnetic flux density

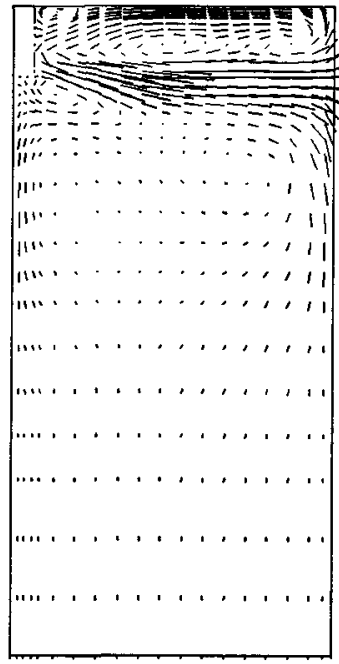

$B \max ($ tesla $)=0.17$

Table 1. Gharacteristics of molten steel.

\begin{tabular}{ll||ll}
\hline$\rho\left(\mathrm{kg} / \mathrm{m}^{3}\right)$ & 7100 & $k(\mathrm{kcal} / \mathrm{m} \cdot \mathrm{s} \cdot \mathrm{k})$ & 0.0111 \\
$\mu(\mathrm{kg} / \mathrm{m} \cdot \mathrm{s})$ & 0.006 & $-\Delta H(\mathrm{kcal} / \mathrm{kg})$ & 64.0 \\
$\sigma(1 / \Omega \cdot \mathrm{m})$ & $7.14 \times 10^{5}$ & $T_{b}\left({ }^{\circ} \mathrm{C}\right)$ & 1525 \\
$C_{p}(\mathrm{kcal} / \mathrm{kg} \cdot \mathrm{k})$ & 0.15 & $T_{s}\left({ }^{\circ} \mathrm{C}\right)$ & 1475 \\
\hline
\end{tabular}

Table 2. Heat transfer coefficient.

\begin{tabular}{lccc}
\hline Region & Free surface & Mold & Spray \\
\hline$U_{t}\left(\mathrm{kcal} / \mathrm{m}^{2} \cdot \mathrm{s} \cdot \mathrm{k}\right)$ & 0.013 & 0.35 & 0.30 \\
\hline
\end{tabular}

for an even inflow of powder between the solidified shell and the mold.

\section{Conclusion}

In order to clarify the effect of EMBR on the heat transfer and the fluid flow in the continuous casting mold, a three dimensional mathematical model has been developed. The following results are obtained.

(1) The agreement appears to be quantitative

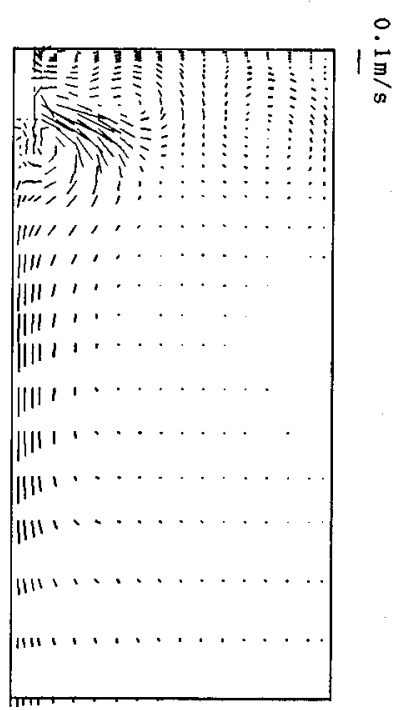

0.35
Fig. 9.

Effect of the position of EMBR on flow field. (at $1 / 4$ thickness of the slab)

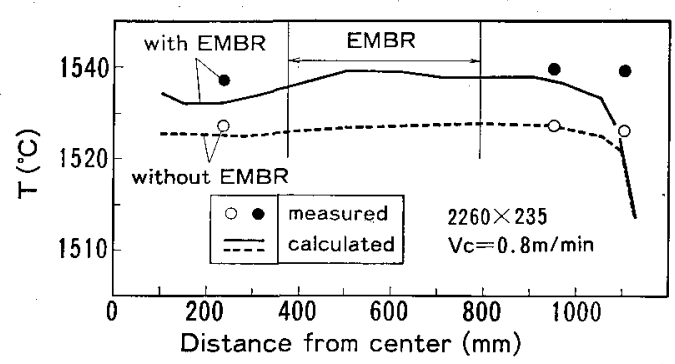

Fig. 10. Comparison of temperature profile at $35 \mathrm{~mm}$ below the meniscus between calculated and measured results. (at $1 / 2$ thickness of the slab)

without any kind of turbulent model by means of dividing the grid into small parts near the mold wall.

(2) It is possible to control the fluid flow by a couple of electromagnet placed at the outlet of the immersion nozzle.

(3) The tcmperature near the meniscus is higher at $1 / 2$ thickness of the slab and the temperature gradient at the meniscus is steeper with EMBR than without EMBR. 


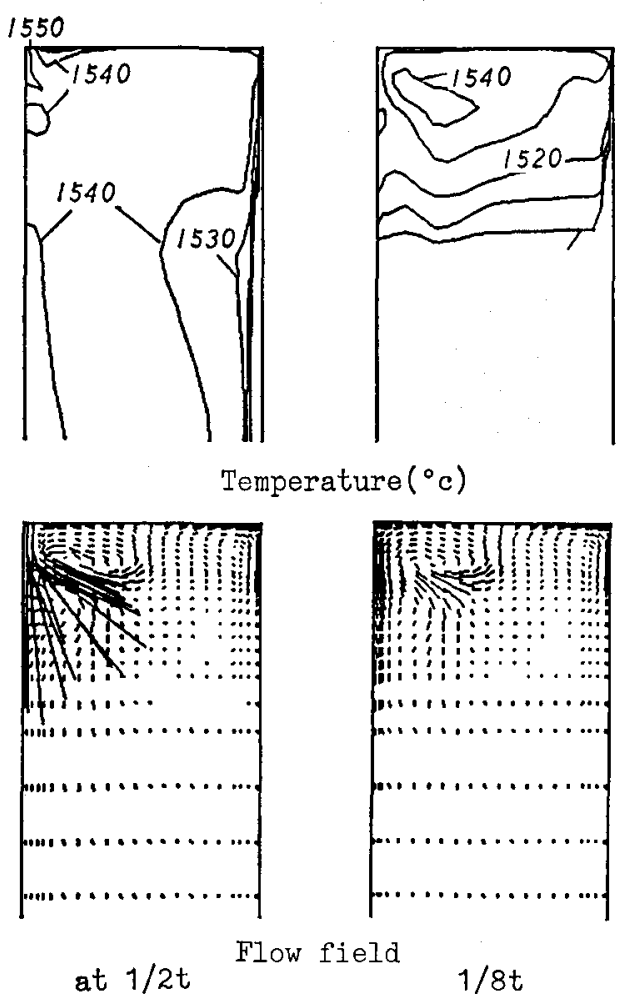

a) With EMBR

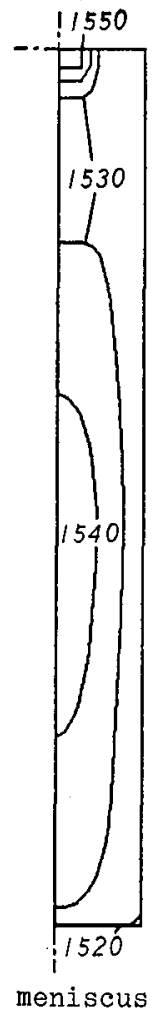

Fig. 11. Temperature profiles at $1 / 2$ and $1 / 8$ thicknesses of the slab and the meniscus.

\section{Nomenclature}

B: Magnetic flux density vector

$C_{p}$ : Specific heat

$\boldsymbol{E}:$ Electric field vector

$f_{s}$ : Fraction solid

$f_{l}$ : Fraction liquid $\left(1-f_{s}\right)$

g: Acceleration vector of gravity

$\mathbf{J}$ : Current density vector

$k$ : Thermal conductivity

$P$ : Pressure

T: Temperature

$T_{l}$ : Liquidus temperature

$T_{s}$ : Solidus temperature

$\mathbf{U}_{l}$ : Velocity vector of liquid phase

$\mathbf{U}_{s}$ : Velocity vector of solid phase

$\mathbf{U}_{t}$ : Heat transfer coefficient

$-\Delta H:$ Latent heat of fusion

$\beta$ : Coefficient of thermal expansion

$\mu:$ Viscosity

$\rho$ : Density

$\sigma:$ Electric conductivity

\section{REFERENCES}

1) K. Hosotani, T. Saitoh, H. Nakato, T. Nozaki, K. Sorimachi and H. Okuda: Tetsu-to-Hagané, 73 (1987), S1446.

2) K. Kinoshita, K. Hosotani, Y. Oguchi, K. Sorimachi, T. Kayano and M. Yao: The Fourth Japan-Nordic Countries Joint Symposium on Science and Technology of Process Metallurgy, ISIJ, Tokyo, (1986), 170.

3) M. Yao, M. Ichimiya, S. Kiyohara, K. Suzuki, K. Sugiyama and R. Mesaki: Steelmaking Proceedings, Vol. 68, ISS-AIME, Penn., (1985), 27.

4) D. D. Gray and A. Giorgini: Int. J. Heat Mass Transfer, 19 (1976), 545.

5) W. H. Hughes and F.J. Young: The Electromagnetodynamics of Fluid, John Wiley \& Sons, Inc., New York, (1966), 148.

6) M. L. Wilkins: Calculation of Elastic--Plastic Flow, Lawrence Radiation Lab., Livermore, (1969), UCRL-7322 Rev. 1, 35.

7) K. Takatani: Tetsu-to-Hagané, 74 (1988), 1546.

8) G. W. Hirt, B. D. Nichls and N. C. Romero: SOLA-A Numerical Solution Algorithm for Transient Fluid Flows, Los Alamos Sci. Lab., Los Alamos, (1975), LA-5852.

9) I. Ohnaka and T. Fukusako: Trans. Iron Steel Inst. Jpn., 17 (1977), 410.

10) 1. Ohnaka and K. Kobayashi: Trans. Iron Steel Inst. Jpn., 26 (1986), 781. 